\title{
Relación entre factores personales y el desempeño académico de estudiantes universitarios en lengua extranjera
}

\author{
Personal factors that affect foreign language academic performance in \\ university students
}

\section{Relação entre fatores pessoais e desempenho acadêmico de estudantes universitários em língua estrangeira}

\author{
Jiménez Rivas José Ramón \\ Universidad Autónoma de la Ciudad de México, México \\ ramon.jimenez@uacm.edu.mx \\ https://orcid.org/0000-0002-4367-7367 \\ Morita Alexander Adelina \\ Universidad Autónoma de Querétaro, México \\ amorita@uaq.edu.mx \\ https://orcid.org/0000-0002-8722-233X
}

\section{Resumen}

Desde hace muchos años, los factores que afectan el desempeño académico han sido estudiados en el ámbito interescolar, local, estatal, nacional e internacional, tanto en instituciones públicas como privadas. Determinar cuál de ellos prevalecen en los estudiantes con mejores logros podría coadyuvar a una mejor enseñanza. Existen indagaciones enfocadas en cómo los factores personales influyen en el aprendizaje; sin embargo, hay poca literatura acerca de su impacto en el desempeño de lengua extanjera. Por tanto, este trabajo se centró en estudiar si algún factor personal (edad, género, o perfil de autodirección del estudiante) guardaba relación con el desempeño académico en la materia Lengua Extranjera, que pertenece al currículo del primer año de una universidad pública localizada al norte de la Ciudad de México. El estudio tuvo un enfoque cuantitativo y un diseño de investigación 


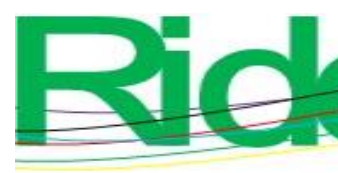

Revista Iberoamericana para la Investigación y el Desarrollo Educativo ISSN 2007-7467

transversal, descriptivo y correlacional. La muestra elegida por conveniencia estuvo conformada por 39 estudiantes de ambos sexos. El instrumento utilizado para medir el perfil de autodirección fue el cuestionario de indagación de perfil autodirigido. Dicho instrumento consiste en 50 ítems, con escala Likert de cinco puntos, y con confiabilidad de 0.90 en el índice alfa de Cronbach. Con los factores personales seleccionados, la edad de los estudiantes, mayoritariamente adultos jóvenes (mujeres, 24.3 años; hombres, 25.92 años) se encontró un coeficiente de correlación de Spearman $r_{s}=.101, p<.540 \mathrm{~N}=39$. Con el género (la muestra estuvo conformada por $66.7 \%$ mujeres y $33.3 \%$ hombres), la correlación de Pearson fue $r=-.387, p<.015 \mathrm{~N}=39$. Finalmente, pese a que los participantes tuvieron un muy buen perfil de autodirección, según el instrumento utilizado, la correlación indicó $\mathrm{r}_{\mathrm{s}}=-$ $.054, p<.744 \mathrm{~N}=39$. A partir del análisis estadístico, se demostró que la interdependencia entre los mencionados factores personales y el desempeño académico es estadísticamente no significativa.

Palabras clave: diferencia individual, enseñanza de una segunda lengua, enseñanza superior, rendimiento escolar.

\section{Abstract}

Academic achievement has been studied for many years in both private and public educational institutions and in local, state, nationwide and international settings. Determining which factors foster academic achievement could enhance learning. There are several studies regarding how personal factors influence learning. Nevertheless, few studies deal with personal factors and academic achievement in foreign language learning. This study aimed to determine whether a personal factor such as age, genre or self-direction fosters language learning academic performance in first year college students. The research used a cuantitative, transversal, descriptive and correlational design. 39 students of a public university in northern Mexico City participated in the study. Most of the participants were young women (66.7\% aged 24.3 on average). Self-directed profile was measured with the Profile Inquiry Self-direction Questionnaire. This tool consists of 50 five-point-Likert-scale items and its Cronbach's alpha equals 0.90. Statistical analysis showed that despite an overall adequate self-direction profile of the sample and the better grades of male students who in turn were older than their female counterparts, language learning academic performance and 


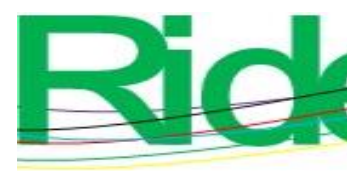

Revista Iberoamericana para la Investigación y el Desarrollo Educativo ISSN 2007 - 7467

personal factors, age $\left(\mathrm{r}_{\mathrm{s}}=.101, \mathrm{p}<.540 \mathrm{~N}=39\right)$; genre $(\mathrm{r}=-.387, p<.015 \mathrm{~N}=39)$ and selfdirection profile $\left(\mathrm{r}_{\mathrm{s}}=-.0540, p<.7440 \mathrm{~N}=39\right)$, had no correlation.

Keywords: individual differences, language instruction, higher education, student achievement.

\section{Resumo}

Por muitos anos, os fatores que afetam o desempenho acadêmico têm sido estudados nos níveis interescolar, local, estadual, nacional e internacional, tanto em instituições públicas como privadas. Determinar qual deles prevalece entre os alunos com melhor desempenho pode contribuir para um ensino melhor. Existem investigações focadas em como os fatores pessoais influenciam a aprendizagem; no entanto, há pouca literatura sobre seu impacto no desempenho de língua estrangeira. Portanto, este trabalho teve como objetivo estudar se algum fator pessoal (idade, gênero ou perfil de autodireção do aluno) estava relacionado ao desempenho acadêmico na disciplina Língua Estrangeira, que pertence ao currículo do primeiro ano de uma universidade pública localizada a norte de Cidade do México. O estudo teve abordagem quantitativa e delineamento de pesquisa transversal, descritiva e correlacional. A amostra escolhida por conveniência foi composta por 39 alunos de ambos os sexos. O instrumento utilizado para mensurar o perfil autodirigido foi o questionário de inquérito de perfil autodirigido. Esse instrumento é composto por 50 itens, com escala Likert de cinco pontos e confiabilidade de 0,90 no índice alfa de Cronbach. Com os fatores pessoais selecionados, a idade dos alunos, em sua maioria adultos jovens (mulheres, 24,3 anos; homens, 25,92 anos), encontrou-se um coeficiente de correlação de Spearman rs =0,101, p $<0,540 \mathrm{~N}=39$. Com o sexo (a amostra foi composta por $66,7 \%$ de mulheres e $33,3 \%$ de homens), a correlação de Pearson foi $\mathrm{r}=-.387, \mathrm{p}<.015 \mathrm{~N}=39$. Por fim, apesar dos participantes apresentarem um perfil de autodireção muito bom, dependendo do instrumento utilizado, a correlação indicou $r s=-0,054, p<0,744 \mathrm{~N}=39$. A partir da análise estatística, evidenciou-se que a interdependência entre os fatores pessoais citados e o desempenho acadêmico não é estatisticamente significativa.

Palavras-chave: diferença individual, ensino de segunda língua, ensino superior, desempenho escolar. 


\section{Introducción}

La teorización sobre los factores que afectan el desempeño académico de los estudiantes comenzó el siglo pasado, cuando Coleman (1966) realizó estudios en diversas escuelas de EE. UU. Su hallazgo: la escuela perpetúa el estado actual de la sociedad. Por ejemplo, los alumnos que provienen de familias con empleos bien remunerados y con una educación de calidad tienen mayores posibilidades de obtener, al término de sus estudios, un mejor trabajo (Atteberry y McEachin, 2020). En la actualidad, y después de años de investigación sobre este tema, los estudios han evolucionado hacia la búsqueda de una correlación que explique, mas allá del factor escolar, cómo el desempeño académico se ve afectado por variables de tipo personal, familiar o contextual (Heredia y Calderón, 2014).

La mayoría de estos trabajos tienen un diseño transeccional (Brown, 2011; Cerda, Parra, López-Vargas y Sáiz, 2014; Yip, 2012; Zavala y Camacho, 2014), y pocos se basan en el diseño longitudinal (Cassidy, 2012; Escamilla y Heredia, 2019; Herrera, Camacho y Heredia, 2016). Además, relacionan uno o dos factores con el desempeño académico en un ciclo escolar, o con una sola materia. Por ejemplo, el perfil de autodirección medido con el instrumento empleado en este estudio (Cipa+) se ha asociado con el desempeño académico y con estilos de aprendizaje en estudiantes de música (Herrera, Camacho y Heredia, 2016); con habilidades de pensamiento en estudiantes normalistas (Escamilla y Heredia, 2019) y con estudiantes de nivel medio superior (Villa y Zamudio, 2014).

En el ámbito de la enseñanza de lenguas extranjeras en educación superior, existen muy pocas indagaciones que relacionen factores personales con el desempeño de los estudiantes. En este rubro, las investigaciones muestran diferentes métodos de evaluación de desempeño: dictados y ejercicios de opción múltiple (Ferrari y Palladino, 2012); calificación de un examen de comprensión de textos (Lafontaine y De Serres, 2007); evaluación de cuatro habilidades con énfasis en la lectura (Lafontaine, 2008), y promedios finales en la materia de lengua extranjera y promedio acumulado (Brown, 2013). Asimismo, los factores personales seleccionados varían de una investigación a otra: perfiles de autorregulación (Elvira-Valdés y Pujol, 2012); edad, género y logro previo (Cassidy, 2012); género y uso de habilidades de autodirección (Cerda et al., 2014); perfil de autoeficacia y estrategias de aprendizaje (Yip, 2012); motivación (Mohammadi, Moenikia y Zahed-Babelan, 2010), etc. El tamaño de las muestras seleccionadas también varía considerablemente: 780 estudiantes de preparatoria, y entre 284 (Diab, 2006) y 30 (Brown, 2013) cuando se trata de estudiantes universitarios. 


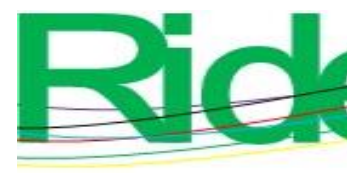

Revista Iberoamericana para la
Investigación y el Desarrollo Educativo
ISSN $2007-7467$

De acuerdo con la Asociación Nacional de Universidades e Instituciones de Educación Superior [Anuies] (2019), un gran número de universidades nacionales cuentan con algún centro de idiomas, mediateca o curso de inglés o francés básico dentro de sus planes curriculares o extracurriculares que tienen la finalidad de coadyuvar al desempeño académico, laboral y/o profesional. De hecho, la acreditación de un idioma extranjero se ha convertido en requisito indispensable en la mayoría de ellas para la titulación de licenciatura y posgrado. Es importante, entonces, distinguir qué factores afectan el desempeño en el aprendizaje de una lengua extranjera.

Por tal motivo, el presente trabajo se enfocó en analizar si alguna variable de tipo personal de los estudiantes (p. ej., edad, sexo o perfil de autodirección) influye en su desempeño académico en los cursos de lengua extranjera en una universidad pública del norte de la Ciudad de México. La pregunta que dirigió el presente estudio fue la siguiente: ¿existe relación entre el desempeño académico en la materia de lengua extranjera y los factores como género, edad o perfil de autodirección de estudiantes que cursan el primer año de licenciatura en una universidad pública?

\section{Hipótesis}

Factores personales tales como la edad, el género o el perfil de autodirección en estudiantes universitarios de primer año no influyen en el desempeño académico de la materia de lengua extranjera.

\section{Objetivos}

El objetivo general fue describir la relación entre el desempeño académico en la materia de lengua extranjera y los factores como género, edad y/o perfil de autodirección de estudiantes que cursan el primer año de licenciatura en una universidad pública. Asimismo, los objetivos particulares fueron los siguientes:

- Describir los factores personales presentes en los alumnos con mejor promedio en los cursos de lengua extranjera.

- Describir el estado del desempeño académico en los cursos de lengua extranjera en la multicitada universidad. 


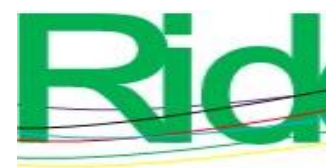

Revista Iberoamericana para la
Investigación y el Desarrollo Educativo ISSN $2007-7467$

\section{Metodología}

Este estudio tuvo un enfoque cuantitativo, y un diseño de investigación transversal, descriptivo y correlacional. El enfoque cuantitativo se caracteriza por la observación estructurada; su objetivo es probar de manera empírica, objetiva y sistemática la relación entre variables por medio de métodos estadísticos (Hernández, Fernández y Baptista, 2014). En este caso, se empleó un instrumento de 50 reactivos, divididos en cinco componentes con escala Likert de cinco puntos. Además, el desempeño académico, así como la edad y el perfil de autodirección de los estudiantes son variables que se pueden cuantificar por medio de escalas numéricas.

Un diseño descriptivo indica que las variables se miden de manera individual y son documentadas. Es descriptivo debido a que se planteó una matriz que incluye los datos de cada estudiante para caracterizarlos plenamente (edad, sexo, carrera, calificaciones en la materia de lengua extranjera, etc.).

Se trata también de un estudio transversal debido a que los datos se recopilaron en un momento único de tiempo (primer semestre del año en curso). Finalmente, esta investigación fue de tipo correlacional porque se procuró describir la relación entre variables por medio con dos métodos estadísticos: la correlación de Pearson y la correlación de Spearman.

\section{Contexto sociodemográfico}

El estudio involucró a estudiantes universitarios de licenciatura de una universidad pública de uno de sus planteles que se encuentra al norte de la Ciudad de México. Los alumnos pertenecen a estratos sociales que van desde escasos recursos hasta posición económica media. La materia Lengua Extranjera se cursa durante el primer año de sus estudios de licenciatura como una asignatura de tronco común o ciclo básico; sin embargo, muchos postergan estos cursos para tomar materias de su especialidad de semestres más avanzados o ciclo superior.

\section{Población y muestra}

La muestra fue elegida por conveniencia, es decir, basada en la oportunidad, beneficio y comodidad (Cohen, 2018). Se seleccionó de esta forma debido a que solo se consideraron los datos de los estudiantes que ya hubieran cursado la materia Lengua Extranjera, que voluntariamente decidieron colaborar en la investigación y que estaban inscritos en el plantel 


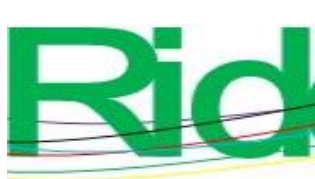

Revista Iberoamericana para la Investigación y el Desarrollo Educativo ISSN 2007 - 7467

de adscripción del autor de este trabajo. La muestra fue de 39 estudiantes de licenciatura (26 mujeres y 13 hombres).

\section{Participantes}

Los 39 estudiantes de la muestra pertenecían a una de las quince carreras que ofrece la universidad; son alumnos activos que ya habían aprobado los tres cursos de la materia Lengua Extranjera en algún semestre anterior a la realización del estudio. La mencionada muestra estuvo conformada por $66.7 \%$ mujeres y $33.3 \%$ hombres, cuyas edades oscilaban entre los 19 y los 64 años.

Un dato interesante, si bien no concurrente en la pregunta de investigación, es el área disciplinar de los participantes: la mayoría (97.4\%) pertenece al área de humanidades y ciencias sociales (tabla 1).

Tabla 1. Caracterización de los participantes por carrera

\begin{tabular}{|l|c|c|}
\hline Carrera & Frecuencia & Porcentaje \\
\hline Arte y patrimonio cultural & 6 & 15.4 \\
\hline Comunicación y cultura & 10 & 25.6 \\
\hline Creación literaria & 3 & 7.7 \\
\hline Ciencia política y administración urbana & 6 & 15.4 \\
\hline Ciencias sociales & 7 & 17.9 \\
\hline Filosofía e historia de las ideas & 3 & 7.7 \\
\hline Historia y sociedad contemporánea & 3 & 7.7 \\
\hline $\begin{array}{l}\text { Ingeniería en sistemas electrónicos y } \\
\text { telecomunicaciones }\end{array}$ & 1 & 2.6 \\
\hline $\mathrm{N}$ & 39 & 100 \\
\hline
\end{tabular}

Fuente: Elaboración propia

\section{Instrumentos}

Las variables utilizadas en este estudio se muestran en la tabla 2. 
Tabla 2. Definición de las variables de estudio e instrumentos para su medición

\begin{tabular}{|c|c|c|c|c|}
\hline Variable & Definición conceptual & $\begin{array}{l}\text { Definición } \\
\text { operacional }\end{array}$ & Instrumento & Ítem \\
\hline Género & $\begin{array}{l}\text { Construcción social de } \\
\text { supuestas diferencias } \\
\text { biológicas entre } \\
\text { hombres y mujeres: sus } \\
\text { roles, ideas, creencias, } \\
\text { representaciones, } \\
\text { atribuciones, etc. }\end{array}$ & $\begin{array}{l}\text { Femenino } \\
\text { Masculino }\end{array}$ & Cuestionario & 2 \\
\hline Edad & $\begin{array}{llr}\text { Cada uno de los } \\
\text { periodos en que } & \text { se } \\
\text { considera dividida } & \text { la } \\
\text { vida humana. }\end{array}$ & Años & Cuestionario & 13 \\
\hline $\begin{array}{l}\text { Autodirecció } \\
\mathrm{n} \text { en el } \\
\text { aprendizaje }\end{array}$ & $\begin{array}{l}\text { Dimensión que se } \\
\text { centra en } \\
\text { preferencia una } \\
\text { estudiante de asumir } \\
\text { responsabilidad en su } \\
\text { aprendizaje. }\end{array}$ & $\begin{array}{l}\text { Escala de } \\
\text { calificación de } 50 \\
\text { a } 250 \text { puntos, } \\
\text { significando los } \\
\text { valores más bajos } \\
\text { en un mejor nivel } \\
\text { de autodirección }\end{array}$ & $\begin{array}{l}\text { CIPA+ } \\
\text { versión } 7\end{array}$ & 50 \\
\hline $\begin{array}{l}\text { Desempeño } \\
\text { académico }\end{array}$ & $\begin{array}{l}\text { Nivel de conocimientos } \\
\text { demostrado en un área o } \\
\text { materia comparado con } \\
\text { la norma de edad y nivel } \\
\text { académico. }\end{array}$ & $\begin{array}{l}\text { Escala numérica } \\
\text { de } 7 \text { a } 10\end{array}$ & $\begin{array}{l}\text { Base de datos } \\
\text { proporcionada } \\
\text { por la } \\
\text { universidad }\end{array}$ & 4 \\
\hline
\end{tabular}

Fuente: Elaboración propia

\section{Perfil de autodirección}

El instrumento utilizado para medir el perfil de autodirección fue el cuestionario de indagación de perfil autodirigido (Cipa+ versión 7), diseñado en 2002 por Cázares (2008, citada por Cázares y Ponce, 2009). La escala de calificación del test fue de 50 a 250 puntos, cuyos valores más bajos indican un mejor perfil de autodirección (Escamilla y Heredia, 2019, p. 18).

En este cuestionario de autovaloración el estudiante califica conductas observables con la frecuencia con que percibe su ocurrencia. Los ítems están distribuidos de forma aleatoria para cada componente (en total 50 afirmaciones). La escala Likert fue del 1 al 5 (1 indica el grado de mayor afinidad y 5 el de menor afinidad). El cuestionario quedó dividido en cinco componentes: 


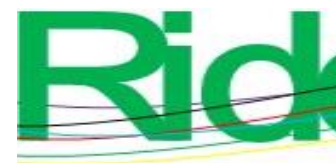

Revista Iberoamericana para la
Investigación y el Desarrollo Educativo
ISSN $2007-7467$

- Componente 1: Planeación y selección de estrategias (10 ítems).

- Componente 2: Autorregulación y motivación (14 ítems).

- Componente 3: Independencia y autonomía (10 ítems).

- Componente 4: Uso de la experiencia y la conciencia crítica (9 ítems).

- Componente 5: Interdependencia y valor social (7 ítems).

El Cipa+ determina además "un valor integrado de los cinco componentes en una escala inversa a los resultados que se determinan como bajo, insuficiente, moderado, muy bueno y óptimo" (Escamilla y Heredia, 2019, p.15). El Cipa+ fue escogido por varias razones, entre las que destacan su disponibilidad, el haber sido utilizado anteriormente en estudios similares y en poblaciones estudiantiles de México y Latinoamérica, la asesoría experta de una de sus desarrolladoras y su confiabilidad de 0.90 en el índice alfa de Cronbach (Cázares y Ponce, 2009); el instrumento fue adaptado para su aplicación en línea con formularios Google.

\section{Base de datos proporcionada por la universidad}

Se solicitaron los datos que pudieran caracterizar a cada uno de los estudiantes de la mejor manera posible: edad, sexo, carrera, calificaciones en la materia Lengua Extranjera de la Universidad Autónoma de la Ciudad de México (UACM, 2019).

Para medir el desempeño académico se tomó el promedio aritmético de los tres semestres de lengua extranjera de cada estudiante. Estos datos fueron facilitados por la Coordinación de registro escolar. La calificación mínima aprobatoria es de 7 y la máxima de 10. También se anotaron las carreras en las que los participantes se hayan inscritos actualmente.

\section{Codificación}

La base de datos se codificó según se muestra en la tabla 3. 
Tabla 3. Codificación de datos

\begin{tabular}{|c|c|c|}
\hline Estudiante & Código & Registro \\
\hline \multicolumn{2}{|l|}{ Edad } & Directo \\
\hline \multirow{2}{*}{ Sexo } & 1 & Femenino \\
\hline & 2 & Masculino \\
\hline \multicolumn{2}{|l|}{ Desempeño } & Promedio aritmético \\
\hline Carrera & $\mathrm{X}$ & Nombre \\
\hline
\end{tabular}

Fuente: Elaboración propia

A partir de la codificación de datos, se hizo el manejo estadístico para ver si existía correlación entre la edad, el sexo y/o el perfil de autodirección con el desempeño académico en la materia Lengua Extranjera.

\section{Resultados}

Al contar con los instrumentos completos, se procedió al análisis vía tabulación de datos y manejo estadístico para encontrar alguna correlación. El procedimiento fue predominantemente descriptivo, es decir, rango, media, mediana, moda, desviación estándar, etc., además de pruebas de correlación como los coeficientes de Pearson y Spearman, sin hacer otra prueba más compleja, pues el número de participantes fue modesto (Okoye y Onokpaunu, 2020). Dichos cálculos se realizaron con el programa computacional estadístico de acceso libre Jamovi (versión 1.2.27).

Los datos obtenidos se presentan de la manera siguiente: primero, los promedios de edad y de calificaciones en la materia de inglés. Segundo, el perfil de autodirección obtenido a partir del uso del instrumento Cipa+. Después se expone el análisis descriptivo del desempeño académico. Finalmente, se muestran las correlaciones de Pearson y Spearman referidas a la edad, sexo y perfil de autodirección con el desempeño académico. En la tabla 4 se observan los promedios de edad y desempeño académico por género. 


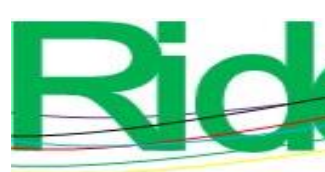

Revista Iberoamericana para la Investigación y el Desarrollo Educativo ISSN $2007-7467$

Tabla 4. Promedio de la calificación de inglés diferenciado por el sexo de los estudiantes

\begin{tabular}{|l|l|l|l|l|}
\hline Sexo & Frecuencia & Porcentaje & $\begin{array}{l}\text { Promedio de } \\
\text { edad } \\
\text { (años) }\end{array}$ & $\begin{array}{l}\text { Promedio de } \\
\text { calificaciones (escala } \\
0-10)\end{array}$ \\
\hline Femenino & 26 & 66.7 & 24.30 & 8.03 \\
\hline Masculino & 13 & 33.3 & 25.92 & 8.59 \\
\hline Total & 39 & 100 & $X$ & $X$ \\
\hline
\end{tabular}

Fuente: Elaboración propia

Se calculó el coeficiente de Pearson presumiendo un comportamiento lineal entre dos variables: una dicotómica (género) y otra continua (desempeño). Sin embargo, el resultado nos muestra que no existe correlación entre el desempeño académico en la materia Lengua Extranjera y el género de los estudiantes (tabla 5).

Tabla 5. Correlación desempeño académico y género

\begin{tabular}{|l|c|}
\hline Correlación de Pearson & Género \\
\hline Coeficiente de correlación r & -.378 \\
\hline Significancia (bilateral) & $<.015$ \\
\hline $\mathrm{N}$ & 39 \\
\hline
\end{tabular}

Fuente: Elaboración propia

Los resultados del perfil de autodirección obtenidos se muestran en las tablas 6 y 7. En la tabla 6, se presentan los resultados desglosados por componente. 


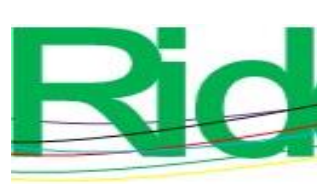

Revista Iberoamericana para la Investigación y el Desarrollo Educativo ISSN 2007 - 7467

Tabla 6. Análisis estadístico descriptivo de los cinco componentes del Cipa+

\begin{tabular}{|l|l|l|l|l|l|l|}
\hline Cipa+ & Rango & Mínimo & Máximo & Media & SD & Varianza \\
\hline Componente 1 & 36 & 9 & 45 & 19.33 & 7.16 & 51.23 \\
\hline Componente 2 & 40 & 10 & 50 & 21.15 & 6.62 & 43.77 \\
\hline Componente 3 & 48 & 12 & 60 & 27.41 & 9.91 & 98.30 \\
\hline Componente 4 & 32 & 8 & 40 & 18.31 & 6.17 & 38.11 \\
\hline Componente 5 & 28 & 7 & 35 & 13.18 & 5.67 & 32.15 \\
\hline Total & 200 & 50 & 250 & 99.38 & 33.06 & 1092.93 \\
\hline
\end{tabular}

$\mathrm{N}=39$

Fuente: Elaboración propia

En la tabla 7 puede apreciarse que un porcentaje alto de participantes $(89.7 \%)$ cuenta con un perfil de autodirección muy bueno u óptimo, considerando la puntuación total.

Tabla 7. Perfil de autodirección de los estudiantes de acuerdo con el puntaje total obtenido

\begin{tabular}{|l|c|c|}
\hline $\begin{array}{l}\text { Integrado de componentes } \\
\text { Cipa+ }\end{array}$ & Frecuencia & \% de la muestra \\
\hline Óptimo 50-90 & 16 & 41.0 \\
\hline Muy bueno 91-130 & 19 & 48.7 \\
\hline Moderado 131-170 & 2 & 5.1 \\
\hline Insuficiente 171-210 & 0 & 0.0 \\
\hline Bajo 211-250 & 2 & 5.1 \\
\hline $\mathrm{N}=39$ & \multicolumn{2}{|}{} \\
\hline
\end{tabular}

Fuente: Elaboración propia

Como se mencionó anteriormente, los datos de las calificaciones obtenidas en los tres cursos de Lengua Extranjera se obtuvieron de la oficina de Registro Escolar. Se calculó el promedio aritmético de los mencionados datos, y estos fueron utilizados en el análisis posterior. Los resultados se presentan en la tabla 8 . 
Tabla 8. Desempeño académico

\begin{tabular}{|l|l|l|}
\hline Frecuencia & Promedio & Porcentaje \\
\hline 1 & 7.0 & 2.6 \\
\hline 6 & 7.3 & 15.4 \\
\hline 5 & 7.7 & 12.8 \\
\hline 7 & 8.0 & 17.9 \\
\hline 9 & 8.3 & 23.1 \\
\hline 5 & 8.7 & 12.8 \\
\hline 1 & 9.0 & 2.6 \\
\hline 2 & 9.3 & 5.1 \\
\hline 3 & 9.7 & 7.7 \\
\hline 39 & 8.33 & 100 \\
\hline Mediana & \multicolumn{2}{|c|}{8.33} \\
\hline Moda & \multicolumn{2}{|c|}{8.33} \\
\hline Varianza & \multicolumn{2}{|c|}{0.843} \\
\hline Desviación estándar & \multicolumn{2}{|c|}{0.918} \\
\hline
\end{tabular}

Fuente: Elaboración propia

En la tabla 9 se muestra la correlación de Pearson de los datos anteriores.

Tabla 9. Correlación desempeño académico con edad

\begin{tabular}{|l|c|}
\hline Correlación de Pearson & Edad \\
\hline Coeficiente de correlación $\mathrm{r}$ & .196 \\
\hline Significancia (bilateral) & $<.233$ \\
\hline $\mathrm{N}$ & 39 \\
\hline
\end{tabular}

Fuente: Elaboración propia

La tabla 10 muestra el desempeño en la materia de inglés y las frecuencias de acuerdo con la edad de cada uno de los participantes. 
Tabla 10. Relación entre rendimiento académico y edad

\begin{tabular}{|l|l|l|l|l|l|l|l|l|l|l|l|l|l|}
\hline & \multicolumn{9}{|c|}{ Edad } \\
\hline Desempeño académico & 19 & 20 & 21 & 22 & 23 & 24 & 25 & 26 & 27 & 28 & 33 & 63 & 64 \\
\hline 7.0 & & 1 & & & & & & & & & & & \\
\hline 7.3 & 3 & & & & & 2 & 1 & & & & & & \\
\hline 7.7 & & 1 & 1 & & & & & 1 & & 1 & & 1 & \\
\hline 8.0 & & 1 & 1 & 2 & 1 & & 1 & & 1 & & & & \\
\hline 8.3 & & 1 & 1 & 2 & 2 & 1 & & 1 & 1 & & & & \\
\hline 8.7 & 2 & 1 & 1 & & & & & & 1 & & & & \\
\hline 9.0 & & & & & & 1 & & & & & & & \\
\hline 9.3 & & & & & & 1 & & & & & 1 & & \\
\hline 9.7 & & 1 & 1 & & & & & & & & & & 1 \\
\hline
\end{tabular}

Fuente: Elaboración propia

Al graficar esta tabla puede apreciarse la dispersión de los datos que nuevamente evidencian la no correlación de tipo lineal entre el desempeño y la edad de los estudiantes. Por simplicidad, en la figura 1 se omitieron los dos últimos valores correspondientes a las edades de 63 y 64 años. 

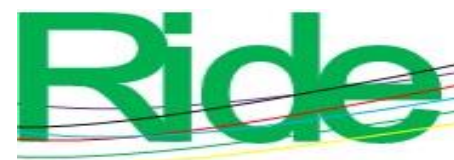

Revista Iberoamericana para la Investigación y el Desarrollo Educativo ISSN 2007 - 7467

Figura 1. Dispersión de datos de edad y desempeño académico

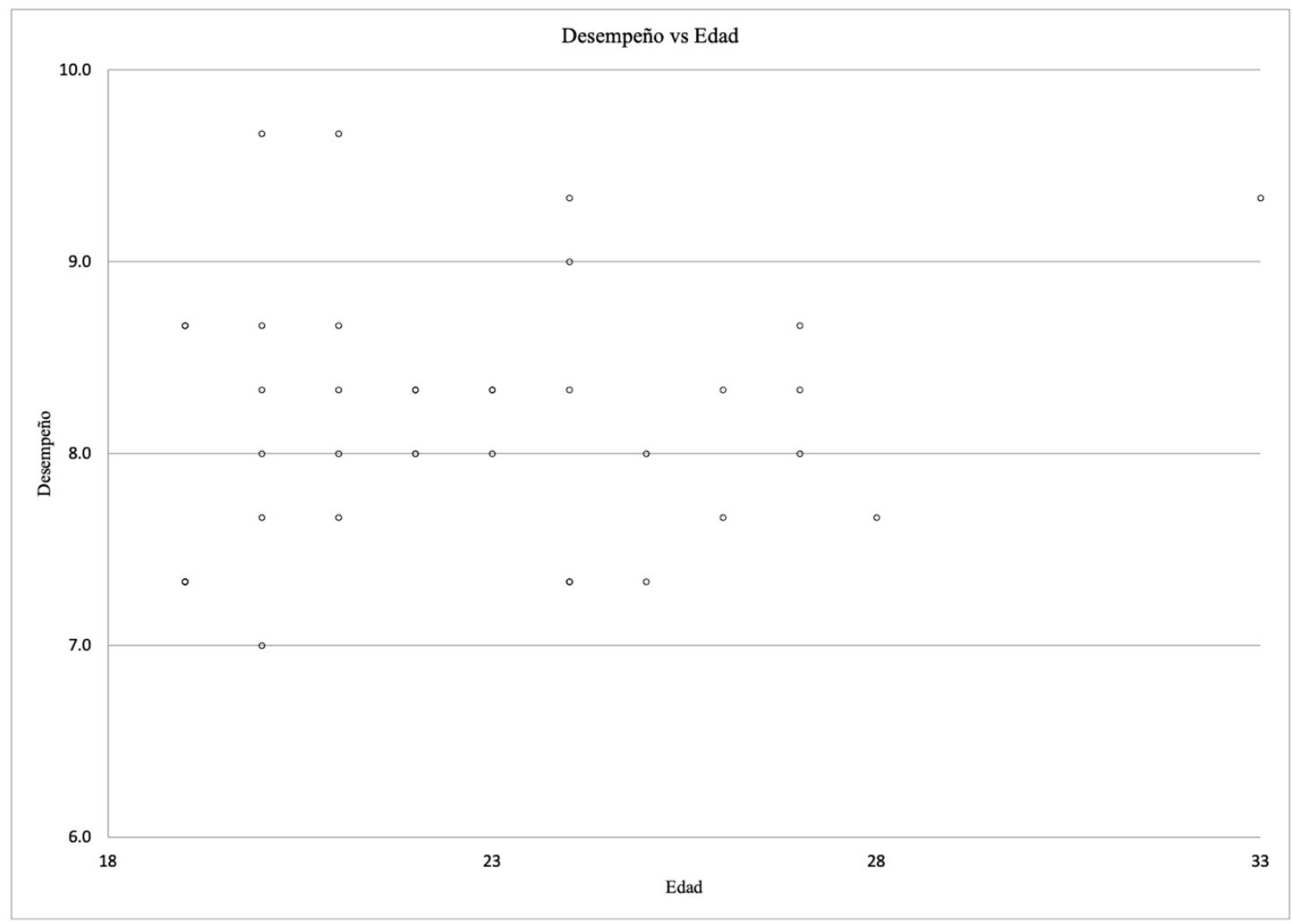

Fuente: Elaboración propia

El análisis de la gráfica muestra una posible relación no-lineal monotónica con pares de variables continuas, por lo que también se calculó el coeficiente de correlación de Spearman. Los resultados se muestran en la tabla 11.

Tabla 11. Correlación desempeño académico con edad

\begin{tabular}{|l|c|}
\hline Correlación de Spearman & Edad \\
\hline Coeficiente de correlación $\mathrm{r}_{\mathrm{s}}$ & .010 \\
\hline Significancia (bilateral) & .540 \\
\hline $\mathrm{N}$ & 39 \\
\hline
\end{tabular}

Fuente: Elaboración propia

En la tabla 12 se muestra el coeficiente de correlación entre el desempeño académico y el perfil de autodirección. 


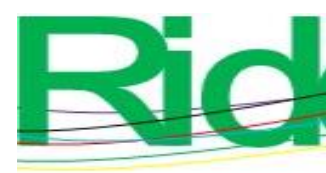

Revista Iberoamericana para la Investigación y el Desarrollo Educativo ISSN $2007-7467$

Tabla 12. Correlación desempeño académico con perfil de autodirección (Cipa+)

\begin{tabular}{|l|c|}
\hline \multicolumn{1}{|c|}{ Correlación de Spearman } & Perfil autodirección \\
\hline Coeficiente de correlación $\mathrm{r}_{\mathrm{s}}$ & -.054 \\
\hline Significancia (bilateral) & .744 \\
\hline $\mathrm{N}$ & 39 \\
\hline
\end{tabular}

Fuente: Elaboración propia

\section{Discusión}

A partir del análisis estadístico, se observa la asiduidad en el desempeño de los estudiantes, ya que las medidas de tendencia central coinciden en un mismo número. Asimismo, el cálculo de la desviación estándar demuestra que este promedio varía en menos de un punto porcentual, por lo que se puede concluir que, en general, el desempeño de estos estudiantes en las materias Inglés y Lengua Extranjera fue bueno.

Del análisis descriptivo, se aprecia que la muestra se compone mayoritariamente por mujeres jóvenes menores de 25 años cuyo desempeño en la materia Inglés fue ligeramente inferior $(6.97 \%)$ que el de su contraparte masculina. Tampoco se aprecia una diferencia significativa en cuanto a la edad en ambos grupos (6.66 \%). La correlación de Pearson (tabla 9) indica que no existe interdependencia de tipo lineal en el desempeño académico en la materia Inglés con la edad de los estudiantes. Esto concuerda con lo hallado por Duque y Martínez (2014), quienes no hallaron relación significativa con esta variable.

En cuanto al género y el desempeño académico, se utilizó la correlación de Spearman debido a que se vincula con los tres criterios básicos de uso (Laerd Statistics, 2020), es decir, dos variables (una continua y otra ordinal). Ambas variables representan pares observados, y finalmente se supuso una relación monotónica entre las variables consideradas. Como se aprecia con el resultado (tabla 5), no existe correlación entre el desempeño académico y el género. Esto concuerda con lo señalado por Elvira-Valdés y Pujol (2012), quienes no encontraron diferencias significativas para la variable género.

En cuanto al análisis del perfil de autodirección y desempeño académico, el cálculo de correlación de Spearman (tabla 12) —entre las calificaciones y el Cipa+ total— no mostró relación alguna. Esto concuerda con Herrera, Camacho y Heredia (2016), quienes no encontraron "una correlación con significación estadística entre el perfil de autodirección y el desempeño académico" (p. 15). 


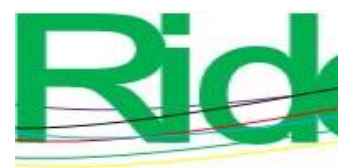

Revista Iberoamericana para la
Investigación y el Desarrollo Educativo
ISSN $2007-7467$

a manera de control para retornar los resultados del cuestionario para el perfil de autodirección (Cipa+) a los participantes del estudio; sin embargo, fue imposible convencer a las diversas instancias burocráticas de lo viable e inocuo de dicha operación.

\section{Conclusiones}

Este trabajo es un estudio exploratorio cuyos datos preliminares constituyen un primer acercamiento para determinar si algún factor personal (edad, género o perfil de autodirección del estudiante) guarda relación con el desempeño académico en la materia Lengua Extranjera, que pertenece al currículo del primer año de una universidad pública localizada en la Ciudad de México. El análisis de los resultados indica que, en el contexto que nos ocupa, el desempeño académico en la materia Lengua Extranjera y los factores personales de los estudiantes son estadísticamente poco significativos.

Empero, es importante que la institución aplique algún instrumento de medición de perfil (autorregulación, autodirección, motivación, habilidades de pensamiento, estilos de aprendizaje, etc.) a estudiantes de primer ingreso. Si bien existe la crítica de que estos instrumentos son el resultado de autoevaluaciones donde se da por hecho que el estudiante contesta a conciencia y con honestidad (pero que en realidad contesta "una cosa pensando en otra", o pensando tal vez que su respuesta sería la de un "buen estudiante"), lo cierto es que los instrumentos ofrecen descripciones de factores observables en el salón de clases que podrían dar a los docentes información adicional para el empleo de actividades conducentes a un aprendizaje más profundo y efectivo.

En este sentido, buscar respuestas a las interrogantes sobre la influencia de factores personales y el desempeño académico, así como su naturaleza en contextos académicos formales o cómo influye en el proceso enseñanza-aprendizaje contribuiría a traspasar prácticas meramente intuitivas o anquilosadas, lo que mejoraría el aprendizaje en el ámbito universitario, en general, y de la enseñanza de lenguas extranjeras, en particular. 


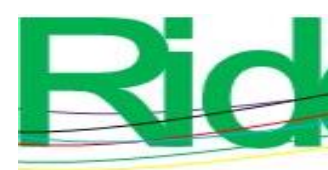

Revista Iberoamericana para la
Investigación y el Desarrollo Educativo
ISSN $2007-7467$

\section{Futuras líneas de investigación}

Las siguientes recomendaciones se realizan con el propósito de ofrecer a los investigadores algunos escenarios para trabajos futuros. La primera es que se replique este estudio con una muestra mayor, que incluya a los estudiantes activos de todos planteles que integran la multicitada universidad junto con los datos de estudiantes egresados y/o titulados. De ese modo se puede desarrollar un análisis profundo sobre los factores personales seleccionados en este trabajo, lo que permitiría descartar definitivamente dichas variables como factores que afectan el desempeño académico en la materia Lengua Extranjera. También sería interesante incluir otros aspectos fácilmente cuantificables que se podrían evaluar como el perfil socioeconómico (factores familiares) o alguna variable escolar (práctica docente), además de la modalidad de lengua extranjera cursada (inglés o francés).

Por último, otro estudio podría referirse a la motivación de los estudiantes para aprender una lengua extranjera, considerando el resultado de "autoconcepto" (componente 3 del perfil de autodirección Cipa +) que involucra la reflexión autocrítica y el deseo por alcanzar metas de aprendizaje o personales, cuyo análisis mostró una moderada falta de tales características en los participantes. Si bien este estudio evidenció que la edad no es relevante en el desempeño, tal vez lo sea como parte del autoconcepto que tiene al involucrarse en el estudio de una lengua extranjera a nivel básico, es decir; ¿cómo influye la motivación de un estudiante (adulto joven) en el aprendizaje de un idioma extranjero? 


\section{Referencias}

Asociación Nacional de Universidades e Instituciones de Educación Superior [Anuies]. (2019). Anuario estadístico de educación superior. http://www.anuies.mx/informacion-y-servicios/informacion-estadistica-deeducacion-superior/anuario-estadistico-de-educacion-superior

Atteberry, A. C. y McEachin, A. J. (2020). Not Where You Start, but How Much You Grow: An Addendum to the Coleman Report. Educational Researcher, 49(9). https://search.ebscohost.com/login.aspx?direct=true \&db=eoah\&AN=53756860\&sit e=ehost-live

Brown, A. V. (2013). Understanding the Relationship Between Language Performance and University Course Grades. Foreign Language Annals, 46(1), 80-87. https://doi.org/10.1111/flan.12014

Cázares, Y. y Ponce, M. (2009). La medición de la autodirección; comparación de tres instrumentos OCLI, SDLRS y CIPA, para obtener el perfil de estudiantes en entornos virtuales. X Congreso Nacional de Investigación Educativa. Consejo Mexicano de Investigación Educativa, A. $\quad$ C., $\quad$ Veracruz, México. http://www.comie.org.mx/congreso/memoriaelectronica/v10/pdf/area_tematica_07/ ponencias/0459-F.pdf

Cassidy, S. (2012). Exploring individual differences as determining factors in student academic achievement in higher education. Studies in Higher Education, 37(7), 793810. https://doi.org/10.1080/03075079.2010.545948

Cerda, C., Parra, J., López-Vargas, O. y Saiz, J. L. (2014). Género, autodirección del aprendizaje y desempeño académico en estudiantes de pedagogía. Educación y Educadores, 17(1). http://educacionyeducadores.unisabana.edu.co/index.php/eye/article/view/3

Cohen, L. A. (2018). Research methods in education. (8a ed.). Nueva York, Estados Unidos: Routledge.

Coleman, J. S. (1966). Equality of educational opportunity (COLEMAN) Study (EEOS). http://doi.org/10.3886/ICPSR06389.v3

Diab, R. L. (2006). University students' beliefs about learning English and French in Lebanon. System, 34(1), 80-96. https://doi.org/10.1016/j.system.2005.06.014 


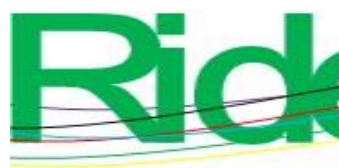

Revista Iberoamericana para la Investigación y el Desarrollo Educativo ISSN $2007-7467$

Dixon, L. Q., Zhao, J., Shin, J.-Y., Wu, S., Su, J.-H., Burgess-Brigham, R. and Snow, C. (2012). What We Know About Second Language Acquisition: A Synthesis From Four Perspectives. Review of Educational Research, 82(1), 5-60. https://doi.org/10.3102/0034654311433587

Duque, C. y Martínez, K. G. (2014). Influencia de los estilos de aprendizaje en el rendimiento académico de los estudiantes de la Facultad de Enfermería de una institución universitaria. En Heredia, Y. y Camacho, D. F. (Comps.), Factores que afectan el desempeño académico (267-291). Monterrey, México: ITESM.

Elvira-Valdés, M. A. y Pujol, L. (2012). Autorregulación y rendimiento académico en la transición secundaria-universidad. Revista Latinoamericana de Ciencias Sociales, Niñez y Juventud, 10(1), 367-378.

Escamilla, M. A. y Heredia, Y. (2019). Autodirección, habilidades de pensamiento y rendimiento académico en estudiantes normalistas. Diálogos sobre Educación, 19(10), 1-6. https://doi.org/10.32870/dse.v0i19.492

Ferrari, M. y Palladino, P. (2012). A Longitudinal Study of English as Foreign Language Learning: L1 Predictors in Italian Students. Applied Cognitive Psychology, 26(4), 616625. https://doi.org/10.1002/acp.2839

Heredia, Y. y Calderón, I. (2014). Factores que afectan el desempeño académico. En Heredia, Y. y Camacho, D. F. (Comps.), Factores que afectan el desempeño académico (11-30). Monterrey, México: ITESM.

Hernández, R., Fernández, C. y Baptista, P. (2014). Metodología de la investigación (6a ed.). Ciudad de México, México: Mc Graw Hill. http://search.ebscohost.com/login.aspx?direct=true \&db=cat02031a\&AN=clase.CLA 01000381473\&lang=es\&site=eds-live

Herrera, K. A., Camacho, D. M. y Heredia, Y. (2016). La relación entre estilos de aprendizaje, autodirección y el desempeño académico en estudiantes de música de una universidad mexicana. Revista Electrónica Complutense de Investigación en Educación Musical, 13, 1-19. http://dx.doi.org/10.5209/RECIEM.51493

Jamovi (1.2.27) (2020). [Computer Software]. The jamovi project. Sidney, Australia: The jamovi proyect. https://www.jamovi.org

Laerd Statistics (Ed.) (2020). Spearman's correlation in SPSS. https://statistics.laerd.com/premium/sroc/spearmans-rank-order-correlation-inspss.php 


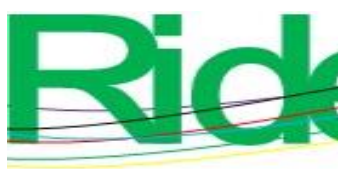
Revista Iberoamericana para la
Investigación y el Desarrollo Educativo
ISSN $2007-7467$

Lafontaine, M. (2008). Validation de mesures d'attitude, de motivation et d'anxiété envers la lecture chez des universitaires francophones. Canadian Journal of Applied Linguistics/Revue Canadienne de Linguistique Appliquée, 11(2), 61-83.

Lafontaine, M. and De Serres, L. (2007). Élaboration et validation d'échelles d'attitudes envers la lecture en francais langue première, de motivation et d'anxiété envers la lecture en anglais langue seconde. Revue canadienne des sciences du comportement, 39(2), 92-109.

Mohammadi, M., Moenikia, M. y Zahed-Babelan, A. (2010). The relationship between motivational systems and second language learning. Procedia - Social and Behavioral Sciences, 2(2), 3258-3262. https://doi.org/10.1016/j.sbspro.2010.03.498

Okoye, K. R. E. y Onokpaunu, M. O. (2020). Relationship between Self-Esteem, Academic Procrastination and Test Anxiety with Academic Achievement of Post Graduate Diploma in Education (PGDE) Students in Delta State University, Abraka. International Scholars Journal of Arts and Social Science Research, 2(3), 37-47.

Schunk, D. H. (2012). Teorías del aprendizaje, una perspectiva educativa (6a ed.). Ciudad de México, México: Pearson.

Universidad Autónoma de la Ciudad de México [Uacm]. (2019). Informe de certificacón de lengua extranjera (2018- 2019). Ciudad de México, México: Uacm.

Villa, M. A. y Zamudio, N. (2014). Los perfiles de autodirección en alumnos de preparatoria con bajo y alto rendimiento académico. En Heredia, Y. y Camacho, D. F. (Comps.), Factores que afectan el desempeño académico (197-218). Monterrey, México: ITESM.

Yip, M. C. W. (2012). Learning strategies and self-efficacy as predictors of academic performance: a preliminary study. Quality in Higher Education, 18(1), 23-34. https://doi.org/10.1080/13538322.2012.667263

Zavala, P. y Camacho, D. F. (2014). Aprendizaje autónomo y rendimiento académico en alumnos de bachillerato. En Heredia, Y. y Camacho, D. F. (Comps.), Factores que afectan el desempeño académico (133-160). Monterrey, México: ITESM. 


\begin{tabular}{|l|l|}
\hline Rol de Contribución & Autor (es) \\
\hline Conceptualización & Jiménez Rivas José Ramón \\
\hline Metodología & Jiménez Rivas José Ramón y Morita Alexander Adelina \\
\hline Software & The jamovi Project \\
\hline Validación & Morita Alexander Adelina \\
\hline Análisis Formal & Jimenez Rivas José Ramón \\
\hline Investigación & Jiménez Rivas José Ramón \\
\hline Recursos & Universidad Autónoma de la Ciudad de México [Uacm] \\
\hline Curación de datos & Jiménez Rivas José Ramón \\
\hline $\begin{array}{l}\text { Escritura - Preparación del } \\
\text { borrador original }\end{array}$ & Jiménez Rivas José Ramón \\
\hline $\begin{array}{l}\text { Escritura - Revisión y } \\
\text { edición }\end{array}$ & Jiménez Rivas José Ramón, Morita Alexander Adelina \\
\hline Visualización & Jiménez Rivas José Ramón \\
\hline Supervisión & NO APLICA \\
\hline Administración de Proyectos & Jiménez Rivas José Ramón \\
\hline Adquisición de fondos & Jivas José Ramón \\
\hline
\end{tabular}

